\title{
Activation of the SphK1/ERK/p-ERK pathway promotes autophagy in colon cancer cells
}

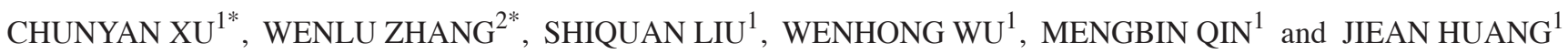 \\ ${ }^{1}$ Department of Gastroenterology, The Second Affiliated Hospital of Guangxi Medical University, Nanning, \\ Guangxi 530007; ${ }^{2}$ Department of Respiratory Medicine, The First Affiliated Hospital of Guangxi Medical University, \\ Nanning, Guangxi 530021, P.R. China
}

Received July 11, 2017; Accepted March 16, 2018

DOI: $10.3892 / \mathrm{ol} .2018 .8588$

\begin{abstract}
Sphingosine kinase 1 (SphK1) is a master kinase that catalyzes the synthesis of sphingosine 1 phosphate and participates in the regulation of cell proliferation and autophagy. The present study aimed to assess the effects of the activation of the SphK1/extracellular signal-regulated kinase (ERK)/phosphorylated (p-)ERK pathway in the regulation of autophagy in colon cancer (HT-29) cells. Inverted fluorescence microscopy was used to detect the expression of green fluorescent protein (GFP) in the SphK1-overexpressing HT-29 cells [SphK1(+)-HT-29] and the negative control HT-29 cells (NC-HT-29). Western blotting was used to detect the protein expression levels of SphK1, ERK1/2, p-ERK1/2, as well as those of the autophagy-associated markers LC3A, ATG5, and ULK1. Protein localization and expression of the LC3A antibody were detected by immunofluorescence. The results demonstrated that GFP was similarly expressed in SphK1(+)-HT-29 and NC-HT-29 cells. However, significantly increased SphK1 mRNA and protein expression levels were detected in SphK1(+)-HT-29 cells compared with in NC-HT-29 cells, which resulted in upregulated ERK/p-ERK. Furthermore, the protein expression levels of the three autophagy-associated markers increased. LC3A protein was localized in the cytoplasm of SphK1(+)-HT-29 cells, indicating autophagy. In summary, the findings of the present study suggested that activation of the SphK1/ERK/p-ERK pathway promotes autophagy in colon cancer HT-29 cells.
\end{abstract}

Correspondence to: Professor Jiean Huang or Professor Shiquan Liu, Department of Gastroenterology, The Second Affiliated Hospital of Guangxi Medical University, 166 Daxuedong Road, Nanning, Guangxi 530007, P.R. China

E-mail: 1404991727@qq.com

E-mail: poempower@163.com

*Contributed equally

Key words: autophagy, sphingosine kinase 1, phosphate-extracellular signal-regulated kinase, colon cancer

\section{Introduction}

Colon cancer is a type of malignant epithelial cell tumor, and presents a major health concern worldwide. The inhibition of cancer cell proliferation is an essential strategy in the treatment of colon cancer (1). However, the molecular mechanisms of colon cancer cell proliferation remain unresolved.

Autophagy is an evolutionarily conserved process in eukaryotes. During autophagy, a nascent double membrane-bound vesicle called an autophagosome encloses a portion of the cytoplasm and the outer membrane of autophagosomes then fuses with the vacuolar or lysosomal membrane to release the inner-membrane structures called autophagic bodies, into the vacuolar or lysosomal lumen for digestion (2). Autophagy serves an important role in the proliferation of colorectal cancer cells (3), and a number of studies have suggested that autophagy prevents metabolites from damaging cells and genomes $(4,5)$. Conversely, other studies have suggested that autophagy contributes to the supply of nutrients and reused metabolites to tumor cells, therefore promoting their survival and proliferation $(6,7)$. Although autophagy has been demonstrated to affect the proliferation of tumor cells, the regulatory mechanism underlying autophagy in colon cancer cells has not been fully investigated.

Sphingosine kinase-1 (SphK1), is an important enzyme that maintains the intracellular sphingolipid balance and has a role in the development of multiple malignancies, plays an important role in resistance to therapies, tumor growth, tumor neovascularization and metastatic spread $(8,9)$. Recently, a study reported that $\mathrm{SphK} 1$ regulates LC3 expression and autophagy in neuroblastoma cells (10). A previous study reported that SphK1 protected the breast cancer cell line MCF-7, induced autophagy and increased cell death from mortality through nutrient starvation (11). Despite the involvement of SphK1 in autophagy, its specific role and associated regulatory mechanism in colon cancer cells remain unclear.

A number of studies have suggested that increased extracellular signal-regulated kinase (ERK) phosphorylation levels induce autophagy in cells $(12,13)$, and that SphK1 promotes the proliferation of colon cancer cells through activation of the ERK/phosphorylated (p-)ERK cascade (14). In the present study, the hypothesis that the activation of the SphK1/ERK/p-ERK pathway promotes autophagy in HT-29 
cells was examined. In order to investigate this, the protein expression levels of SphK1, ERK1/2 and p-ERK1/2, and those of the autophagy-associated markers LC3A, ATG5, and ULK1, were analyzed following the upregulation of SphK1 in HT-29 cells. Additionally, the protein localization and expression patterns of intracellular LC3A, a key marker of autophagy, were assessed.

\section{Materials and methods}

Cell lines and culture. The human colorectal cancer cell line HT-29, Caco-2, RKO and HCT116 cells were purchased from the American Type Culture Collection (Manassas, VA, USA). Cells were cultured in Dulbecco's modified Eagle's medium (Gibco; Thermo Fisher Scientific, Inc., Waltham, MA, USA) supplemented with $10 \%$ fetal bovine serum (FBS; Excell Bio, Inc., Shanghai, China) at $37^{\circ} \mathrm{C}$ with $5 \% \mathrm{CO}_{2}$.

Cell transfection. The Lentiviral vector PLenti-SPHK1IRES-EGFP and the blank vector (NC; R\&S Biotechnology Co., Ltd., Shanghai, China) were used for infection of cancer cells, the cells inoculated with lentivirus at a multiplicity of infection (MOI) of 20 for $48 \mathrm{~h}$, and the percentage of infected cells was approximately $90 \%$ at this MOI. Blasticidins $(2 \mu \mathrm{g} / \mathrm{ml})$ (Merck KGaA, Darmstadt, Germany) was added for 2 weeks. The SphK1-overexpressing HT-29 cells [SphK1(+)-HT-29] and the corresponding negative control HT-29 cells (NC-HT-29) were detected by fluorescence-activated cell sorting. The stabilized transfected SphK1(+)-HT-29 and NC-HT-29 cells were stored in liquid nitrogen (Jinfeng liquid Nitrogen Container Co., Ltd., Chengdu, China) and were used within 3 months between transfection and subsequent experimentation. Cells were cultured in Dulbecco's modified Eagle's medium (Gibco; Thermo Fisher Scientific, Inc., Waltham, MA, USA) supplemented with $10 \% \mathrm{FBS}$ at $37^{\circ} \mathrm{C}$ with $5 \% \mathrm{CO}_{2}$.

Inverted fluorescence microscopy analysis. SphK1(+)-HT-29 and NC-HT-29 cells were seeded onto a 6-well plate and cells covered $\sim 95 \%$ of each well. The cells were observed under an inverted fluorescence microscope (TS100-F; Nikon Corporation, Tokyo, Japan) at x100 magnification. The NIS-Elements software (version 4.0; Nikon Corporation, Tokyo, Japan) was used for cell imaging, according to the manufacturer's protocol. The transfection efficiency of cells was calculated as follows: The number of cells in 3 randomly selected fields that expressed green fluorescent protein (GFP)/the total number of cells.

Reverse transcription-quantitative polymerase chain reaction (RT-qPCR) analysis. RNA isolation was performed using the Total RNA Extraction Kit (Tiangen Biotech Co., Ltd., Beijing, China), according to the manufacturer's protocol. cDNA synthesis was performed using the Reverse Transcription Kit (Takara Bio, Inc., Otsu, Japan). A fluorescence-based qPCR method was performed using $2 \mu \mathrm{l}$ cDNA, $10 \mu \mathrm{l}$ SYBR Green (Takara Bio, Inc.), 0.6 $\mu \mathrm{l}$ PCR forward primer $(10 \mu \mathrm{M}), 0.6 \mu \mathrm{l}$

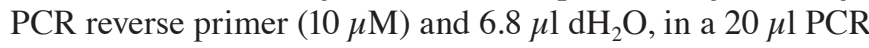
reaction volume. The RT-qPCR reaction was run on a StepOne Real-Time PCR system (Applied Biosystems; Thermo Fisher Scientific, Inc.). The cycling parameters were as follows:
Denaturing at $95^{\circ} \mathrm{C}$ for $30 \mathrm{sec}, 40$ cycles at denaturing at $95^{\circ} \mathrm{C}$ for $5 \mathrm{sec}$, primer annealing at $60^{\circ} \mathrm{C}$ for $34 \mathrm{sec}$, and extension temperature at $95^{\circ} \mathrm{C}$ for $15 \mathrm{sec}$; final extension at $60^{\circ} \mathrm{C}$ for $1 \mathrm{~min}$ and final denaturing at $95^{\circ} \mathrm{C}$ for $15 \mathrm{sec}$. Gene expression levels were determined via the $2^{-\Delta \Delta \mathrm{Cq}}$ method (15), using GAPDH as a reference gene, with the GAPDH gene expression level in NC-HT-29 cells set to 1 . The primers of GAPDH and SphK1 were obtained from Takara Bio, Inc., (Otsu Japan). GAPDH, forward: 5'-GCACCGCAAGGCTGAGAAC-3', and reverse: 5'-TGGTGAAGACGCCAGTGGA-3'; SphK1, forward: 5'-GGCTTCATTGCTGATGTGGA-3', and reverse: 5'-AGGAAGGTGCCCAGAGTGAA-3'.

Western blotting analysis. Total proteins were extracted using radioimmunoprecipitation buffer (Beyotime Institute of Biotechnology, Haimen, China). Protein concentrations were measured by bicinchoninic acid assay (Solarbio Biotech Co., Ltd., Beijing, China) according to the manufacturer's protocol. A total of $30 \mu \mathrm{g}$ of protein from each sample was separated via $12 \%$ SDS-PAGE (Beyotime Institute of Biotechnology, Haimen, China) for $1 \mathrm{~h}$ at $100 \mathrm{~V}$, and then transferred onto nitrocellulose membranes. Samples were blocked with 5\% nonfat-milk in Tris-buffered saline with Tween-20 (Solarbio Biotech Co., Ltd., Beijing, China) for $1 \mathrm{~h}$ at room temperature. The membranes were incubated overnight at $4^{\circ} \mathrm{C}$ with antibodies diluted in WB Antibody Diluent (Beyotime Institute of Biotechnology, Haimen, China). Subsequently, the membranes and secondary antibodies were incubated for $1 \mathrm{~h}$ at room temperature. Bands were quantified by Odyssey infrared imaging (LICOR Biosciences, Lincoln, NE, USA) and GAPDH acted as an internal reference. Rabbit polyclonal anti-SphK1 (dilution 1:1,000; catalog no. A0139), mouse monoclonal anti-ERK1/2 (dilution 1:1,500; catalog no. A10613), rabbit polyclonal anti-p-ERK1/2 (dilution 1:1,500; catalog no. AP0472), mouse monoclonal anti-ATG5 (dilution 1:1,000; catalog no. A2468) and rabbit polyclonal anti-ULK1 (dilution 1:2,000; catalog no. A8529) were purchased from ABclonal, Inc. (Woburn, MA, USA). Rabbit monoclonal anti-LC3A (dilution 1:1,000, 4599) was purchased from Cell Signaling Technology, Inc. (Danvers, MA, USA). Rabbit polyclonal anti-GAPDH (dilution 1:2,000; catalog no. 10494-1-AP) was purchased from ProteinTech Group (Rosemont, IL, USA). The secondary antibodies horseradish-peroxidase (HRP)-conjugated Goat Anti-Rabbit IgG (dilution 1:10,000; catalog no. AS014) and HRP-conjugated Goat Anti-Mouse IgG (dilution 1:10,000; catalog no. AS003) were purchased from ABclonal Inc.

Immunofluorescence. The Cell slide (Solarbio Biotech Co., Ltd., Beijing, China) was placed in 24-well plates and then cells were seeded at a density of $1 \times 10^{5}$. Routinely cultured overnight at $37^{\circ} \mathrm{C}$ with $5 \% \mathrm{CO}_{2}$, the cells were washed with $\mathrm{PBS}$, fixed in a $4 \%$ paraformaldehyde solution (Solarbio Biotech Co., Ltd., Beijing, China ) for $20 \mathrm{~min}$, permeabilized with $0.5 \%$ Triton $\mathrm{X}-100$ for $10 \mathrm{~min}$, sealed with $10 \%$ FBS diluted with $10 \%$ PBS (Excell Bio, Inc., Shanghai, China) for $20 \mathrm{~min}$, and then incubated overnight at $4^{\circ} \mathrm{C}$ with rabbit polyclonal anti-LC3A (dilution 1:500; catalog no. 4599, CST, USA). Subsequently, the cells were incubated with Anti-Rabbit IgG Fab2 Alexa Flour ${ }^{\circledR} 594$ (dilution 1:500; catalog no. 8889S; 

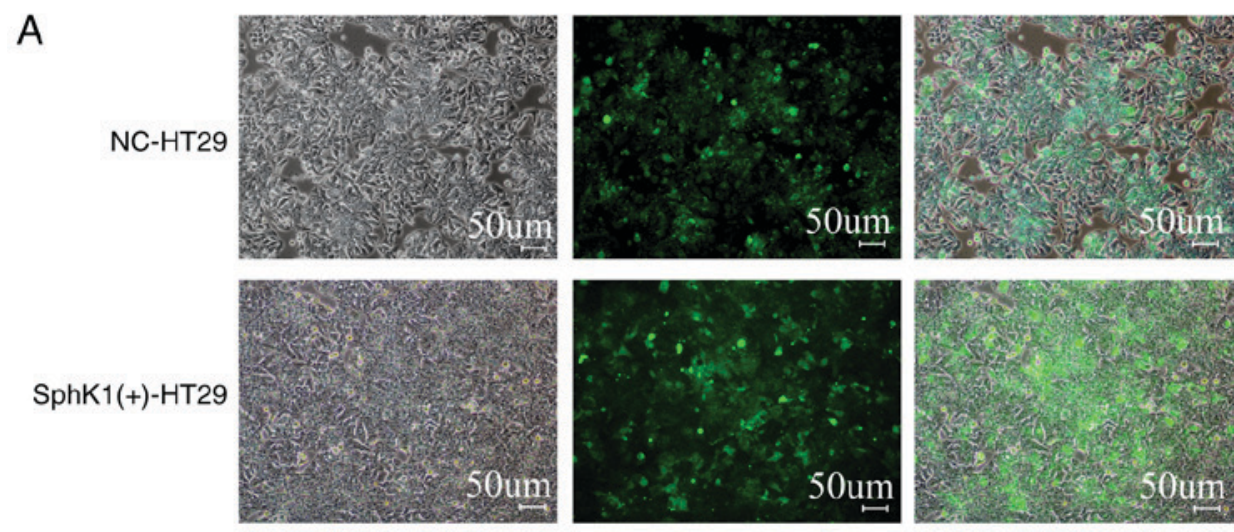

Visible

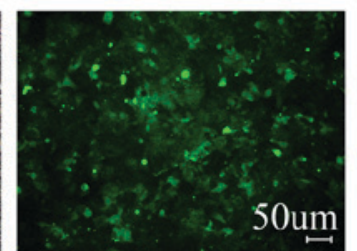

Fluorescence

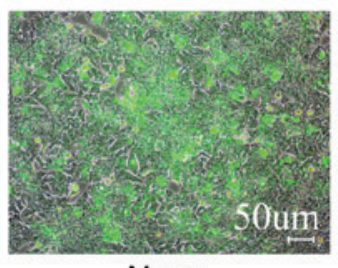

Merge

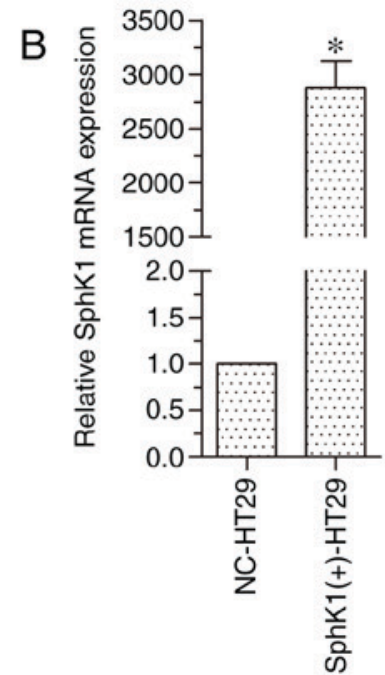

Figure 1. (A) SphK1(+)-HT-29 and NC-HT-29 cells were observed by fluorescence microscopy (magnification, x100). (B) A histogram illustrating the relative expression of SphK1 mRNA in SphK1(+)-HT-29 and NC-HT-29 cells. Expresison in NC-HT-29 cells was set to 1 . (*P<0.05 vs. NC-HT-29). SphK1, sphingosine kinase 1 ; NC, negative control.

Cell Signaling Technology, Inc.) for $1 \mathrm{~h}$ at $37^{\circ} \mathrm{C}$. Cells were stained with DAPI (Beyotime Institute of Biotechnology) for $1 \mathrm{~min}$, and then covered with anti-fluorescent quenching fluid (Beyotime Institute of Biotechnology). An Olympus BX53 (Olympus Corporation, Tokyo, Japan) polarizing microscope was used to observe the cells under x600 magnification and obtain images for further analysis.

Statistical analysis. Each immunofluorescence assay was performed a minimum of three times. Statistical analysis was based on the unpaired Student's t test or the one-way analysis of variance test using SPSS v.16.0 software (SPSS, Inc., Chicago, IL, USA). Data are presented as the mean \pm standard deviation. $\mathrm{P}<0.05$ was considered to indicate a statistically significant difference.

\section{Results}

SphK1 expression is upregulated in transfected HT-29 cells. In a previous study, the relative mRNA expression of SphK1, when compared with that of the reference gene GAPDH, was $0.96 \pm 0.02$ in $\mathrm{Caco} 2$ (colon adenocarcinoma) cells, $0.61 \pm 0.07$ in HT-29 cells, $0.92 \pm 0.05$ in RKO (colon carcinoma) cells and $0.97 \pm 0.02$ in HCT116 cells (16). Therefore, the lowest expression of SphK1 occurred in the HT-29 cell line. To avoid cell autophagy caused by chemical stress, a pLenti-SPHK1-IRES-EGFP vector and a blank vector (NC) were used to transform HT-29 cells in order to obtain an increase in SphK1 expression. The SphK1(+)-HT-29 and NC-HT-29 cells expressed GFP (Fig. 1A) with a transfection efficiency of $92 \%$ in NC-HT-29 cells and $95 \%$ in SphK1(+)-HT-29 cells. RT-qPCR results, using GAPDH as a reference gene and the SphK1 expression level of NC-HT-29 cells set to 1 , demonstrated that the relative expression of SphK1 in SphK1(+)-HT-29 cells was significantly increased (Fig. 1B). These results also indicated that SphK1(+)-HT-29 and NC-HT-29 cells were suitable for the subsequent experiments.
The SphK1/ERK/p-ERK pathway is activated in HT-29 cells. The protein expression levels of SphK1, total ERK1/2 and p-ERK, as detected by western blotting, are illustrated in Fig. 2. SphK1 and p-ERK protein expression was increased in SphK1(+)-HT-29 cells, compared with in NC-HT-29 cells, while there were no significant differences in total ERK1/2 expression. These results suggest that SphK1 activates ERK by phosphorylation.

Autophagy in HT-29 cells is induced by activation of the SphK1/ERK/p-ERK pathway. LC3A protein is a recognized marker for autophagy (17-19), which, upon staining, presents a spotted aggregation pattern under fluorescence microscopy $(20,21)$. As shown in Fig. 3, the protein expression of LC3A appeared as a spotted aggregation in the cytoplasm of SphK1(+)-HT-29 cells, but not in NC-HT-29 cells, suggesting that SphK1 promotes autophagy in HT-29 cells. ATG5 and ULK1 proteins are other important autophagy-associated markers (21-23). In the present study, the protein expression of LC3A, ATG5 and ULK1 was increased in SphK1(+)-HT-29 cells when compared with in NC-HT-29 cells (Fig. 4). Previous studies have reported that increased expression of p-ERK decreases the levels of mTOR/p-mTOR, which then results in increased expression of ULK1 $(24,25)$. When analyzed together, these results suggested that autophagy in HT-29 cells is induced via activation of the SphK1/ERK/p-ERK pathway.

\section{Discussion}

Increasing evidence suggests that the sphingosine kinase-1 (SphK1) serves an important role in the development of cancer, including cell proliferation, apoptosis, metastasis, angiogenesis and chemotherapeutic resistance (9). It has also been reported that $\mathrm{SphK} 1$ promotes the proliferation and metastasis of colon cancer (14). SphK1 is involved in the regulation of sphingolipid metabolism via the production of sphingosine-1 (S1P) $(26,27)$. SphK1/S1P regulates cell proliferation through multiple pathways including the ERK, P38 mitogen-activated 


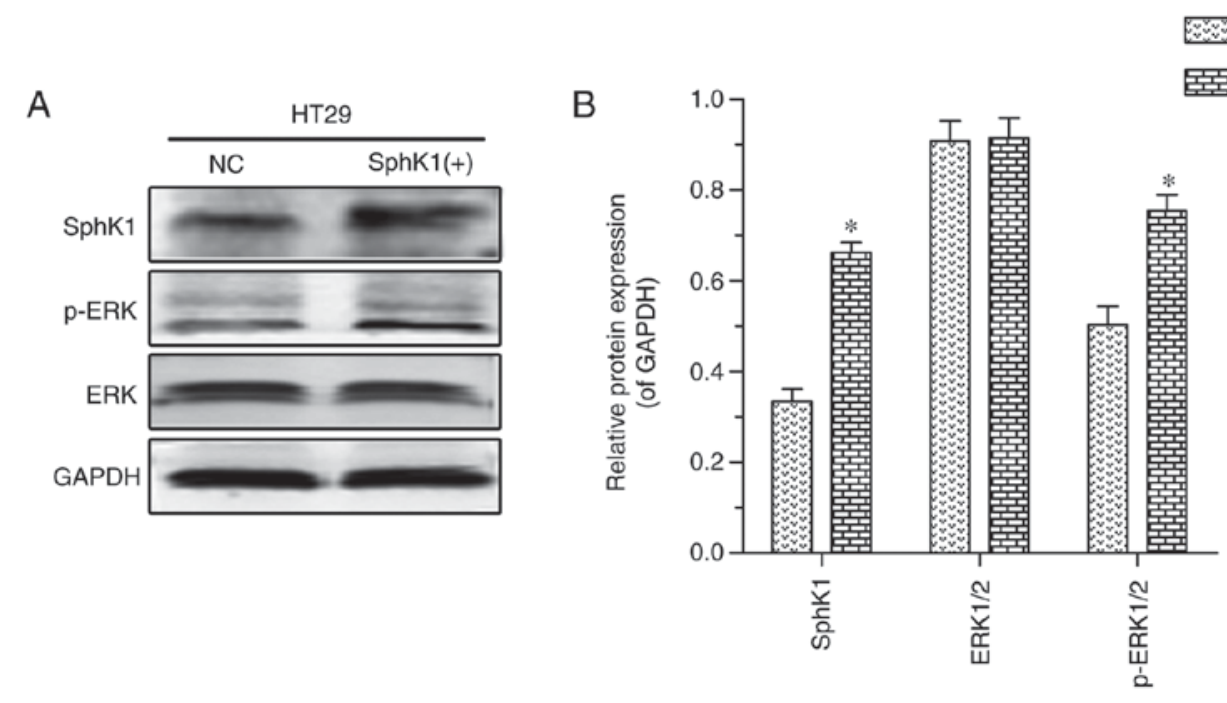

Figure 2. (A) Proteins bands, as obtained by western blotting. (B) Histogram illustrates the protein expression levels of SphK1, ERK1/2 and p-ERK1/2 in SphK1(+)-HT-29 and NC-HT-29 cells. GAPDH was used as the reference gene. Data are presented as the mean \pm standard deviation. ("P<0.05 vs. NC-HT-29). SphK1, sphingosine kinase 1; NC, negative control; p-, phosphorylated; ERK, extracellular signal-regulated kinase.

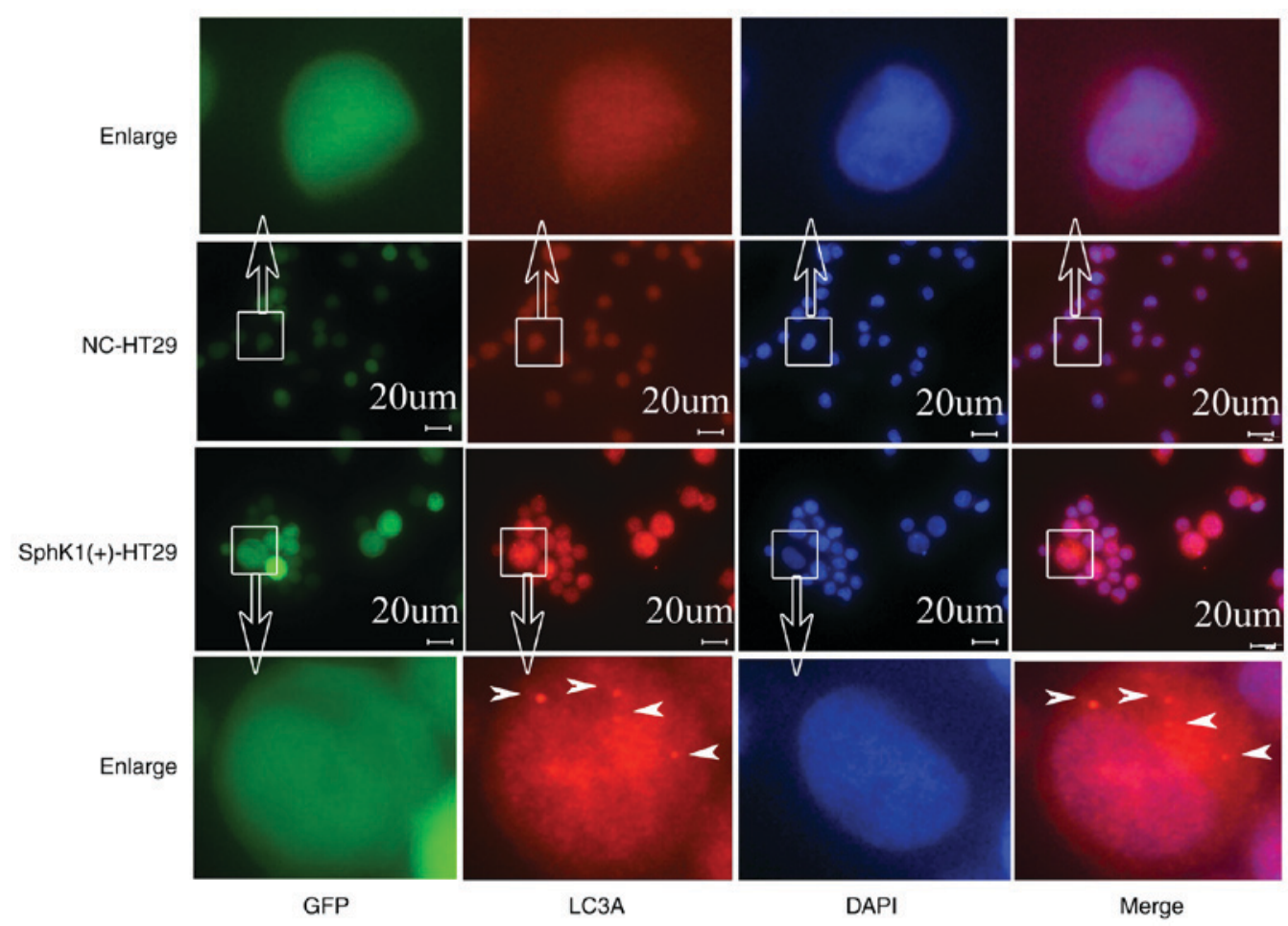

Figure 3. The protein expression of LC3A, as detected by immunofluorescence in SphK1(+)-HT-29 and NC-HT-29 cells (magnification, x600). SphK1, sphingosine kinase 1; NC, negative control; GFP, green fluorescent protein. The white arrows indicate the LC3 protein formed a spotted aggregation pattern.

protein kinase (MAPK) and Akt pathways (28). In the present study, SphK1 expression upregulated ERK phosphorylation, as previously hypothesized. This is consistent with the results of a previous study, which reported that the activation ERK is elevated by the upregulation of SphK1 and attenuated by the suppression of SphK1, while the blocking of the ERK pathway suppressed the effects that are mediated by the overexpression of SphK1 (14). These results suggest that SphK1 modulates the ERK/p-ERK pathway.

ERK is an important component of the MAPK system, which is one of the most important signaling cascades, and has been identified as frequently dysregulated in tumors (29). Additionally, an increase in the expression of ERK and p-ERK led to a decrease in the levels of mTOR and p-mTOR (24). mTOR is an inhibitor of ULK1 (25), which induces the initiation of autophagy (23). Similarly, in the present study, increased levels of p-ERK were observed to promote an increase in ULK1 protein expression. Furthermore, it has been reported that ERK and its upstream kinase MEK are localized in the extra-luminal face of the autophagosomes, and that ERK interacts with autophagy proteins via its substrate-binding domains (20). Overall, 
A

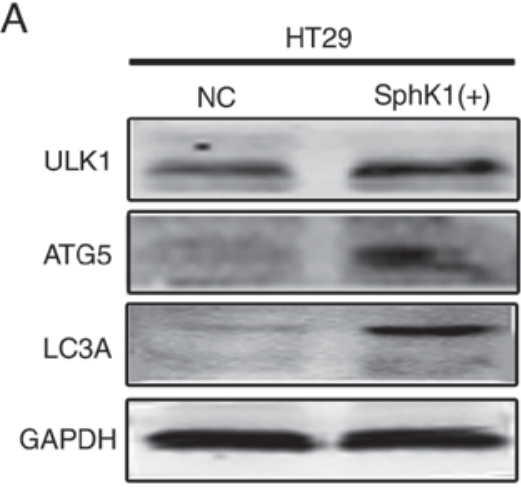

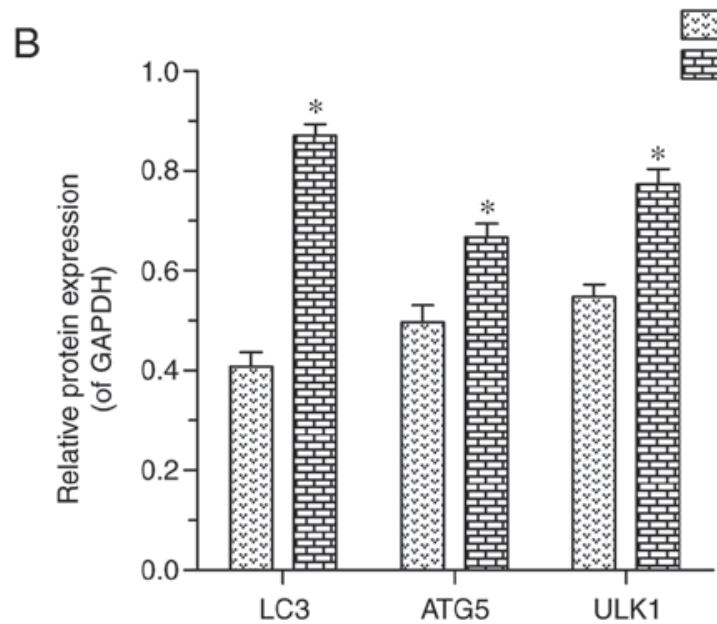

Figure 4. (A) Protein bands, as obtained by western blotting. (B) Quantification of the western blot bands illustrate the protein expression levels of LC3A, ATG5 and ULK1 in SphK1(+)-HT-29 and NC-HT-29 cells. GAPDH was used as the reference gene. Data are presented as the mean \pm standard deviation. ("P<0.05 vs. NC-HT-29). SphK1, sphingosine kinase 1; NC, negative control.

these results suggest that increasing ERK phosphorylation induces cell autophagy $(12,13)$.

Autophagy is a degradation system that supplies cytoplasmic components into the lysosome or vacuole, where the degradation of lipid droplets is known to occur (30). Autophagy eliminates incorrectly translated proteins, metabolic waste and toxic oxygen free-radicals in cancer cells in order to achieve self-renewal and to promote the growth and development of cells (31). Cell autophagy begins with the formation of a bilayer structure termed an autophagosome. Subsequently, two ubiquitination systems are activated, including the ATG8/LC3 phosphatidylethanolamine conjugate system and the ATG12-ATG5 conjugate system (22). LC3 and ATG5 proteins are, consequently, regarded as the principal autophagy-associated proteins. Ubiquitination systems are widely known to be involved in various physiological processes, including cell proliferation, apoptosis and autophagy (32). In the present study, the LC3 protein formed a spotted aggregation pattern in SphK1(+)-HT-29 cells under fluorescent microscopy, which suggested autophagy; this was in accordance with the results of previous studies $(20,21)$. Furthermore, the results of the present study demonstrated that the protein expression of LC3A, ATG5 and ULK1 were increased in the SphK1-upregulated HT-29 cells. These results suggested that SphK1 regulates the expression of LC3 and promotes the autophagy process in colon cancer cells, which is consistent with SphK1 induced autophagy in neuroblastoma and breast cancer cells $(13,14)$.

In summary, the present study demonstrated that activation of the SphK1/ERK/p-ERK pathway promotes autophagy in colon cancer HT-29 cells. An increase in SphK1 lead to an upregulation of ERK/p-ERK by increasing ERK phosphorylation, which in turn resulted in an increase in the expression level of the autophagy-associated markers LC3, ATG5 and ULK1 in SphK1(+)-HT-29 cells. These findings provide a rationale for the development of SphK1 inhibitors, or other cell autophagy inhibitors, as part of a therapeutic strategy for patients with colorectal cancer or other epithelial tumor types. Furthermore, in order to further investigate the role of autophagy in colorectal cancer cells, gene regulation of ERK expression or change cells culture conditions is need in future studies.

\section{Acknowledgements}

Not applicable.

\section{Funding}

This work was supported by grants from the National Natural Science Foundation of China (grant nos. 81460380 and g81260365) and the Innovation Project of Guangxi Graduate Education (grant nos. YCBZ2017035 and YCSW2017100).

\section{Availability of data and materials}

All data generated or analyzed during this study are included in this published article.

\section{Authors' contributions}

CYX and WLZ conceived and designed the study and conducted the experiments. CYX assisted with drafting the manuscript. WHW and MBQ performed the statistical analysis. JAH and SQL interpreted the statistical analysis, reviewed and made final approval of the version to be published. All authors read and approved the manuscript.

\section{Ethics approval and consent to participate}

Not applicable.

\section{Consent for publication}

Not applicable.

\section{Competing interests}

The authors declare that they have no competing interests. 


\section{References}

1. Nagappan A, Lee WS, Yun JW, Lu JN, Chang SH, Jeong JH, Kim GS, Jung JM and Hong SC: Tetraarsenic hexoxide induces G2/M arrest, apoptosis, and autophagy via PI3K/Akt suppression and p38 MAPK activation in SW620 human colon cancer cells. PLoS One 12: e174591, 2017.

2. Izumi $\mathrm{M}$ and Nakamura S: Chloroplast protein turnover: The influence of extraplastidic processes, including autophagy. Int J Mol Sci 19: E828, 2018.

3. Lampada A, O'Prey J, Szabadkai G, Ryan KM, Hochhauser D and Salomoni P: mTORC1-independent autophagy regulates receptor tyrosine kinase phosphorylation in colorectal cancer cells via an mTORC2-mediated mechanism. Cell Death Differ 24: 1045-1062, 2017.

4. He M, Luo M, Liu Q, Chen J, Li K, Zheng M, Weng Y, Ouyang L and Liu A: Combination treatment with fasudil and clioquinol produces synergistic anti-tumor effects in U87 glioblastoma cells by activating apoptosis and autophagy. J Neurooncol 127: 261-270, 2016.

5. Cheng YC, Hueng DY, Huang HY, Chen JY and Chen Y: Magnolol and honokiol exert a synergistic anti-tumor effect through autophagy and apoptosis in human glioblastomas. Oncotarget 7: 29116-29130, 2016.

6. Zhang L, Yu Y, Xia X, Ma Y, Chen XW, Ni ZH and Wang H: Transcription factor E2-2 inhibits the proliferation of endothelial progenitor cells by suppressing autophagy. Int J Mol Med 37: 1254-1262, 2016.

7. Samardzija G, Stevovic TK, Djuricic S, Djokic D, Djurisic M, Ciric D, Martinovic T, Bumbasirevic V and Vujic D: Aggressive human neuroblastomas show a massive increase in the numbers of autophagic vacuoles and damaged mitochondria. Ultrastruct Pathol 40: 240-248, 2016.

8. Long J, Xie Y, Yin J, Lu W and Fang S: SphK1 promotes tumor cell migration and invasion in colorectal cancer. Tumour Biol 37: 6831-6836, 2016.

9. Almejún MB, Borge M, Colado A, Elías EE, Podaza E, Risnik D, De Brasi CD, Stanganelli C, Slavutsky I, Cabrejo M, et al: Sphingosine kinase 1 participates in the activation, proliferation and survival of chronic lymphocytic leukemia cells. Haematologica 102: e257-e260, 2017.

10. Moruno Manchon JF, Uzor NE, Finkbeiner S and Tsvetkov AS: SPHK1/sphingosine kinase 1-mediated autophagy differs between neurons and SH-SY5Y neuroblastoma cells. Autophagy 12: 1418-1424, 2016.

11. Lavieu G, Scarlatti F, Sala G, Carpentier S, Levade T, Ghidoni R, Botti J and Codogno P: Regulation of autophagy by sphingosine kinase 1 and its role in cell survival during nutrient starvation. J Biol Chem 281: 8518-8527, 2006.

12. Ren H, Guo H, Thakur A, Zhang S, Wang T, Liang Y, Shi P, Gao L, Liu F, Feng J, et al: Blockade efficacy of MEK/ERK-dependent autophagy enhances PI3K/Akt inhibitor NVP-BKM120's therapeutic effectiveness in lung cancer cells. Oncotarget 7: 67277-67287, 2016.

13. Zhao Y, Fan D, Zheng ZP, Li ET, Chen F, Cheng KW and Wang M: 8-C-(E-phenylethenyl)quercetin from onion/beef soup induces autophagic cell death in colon cancer cells through ERK activation. Mol Nutr Food Res 61, 2017.

14. Liu SQ, Huang JA, Qin MB, Su YJ, Lai MY, Jiang HX and Tang GD: Sphingosine kinase 1 enhances colon cancer cell proliferation and invasion by upregulating the production of MMP-2/9 and uPA via MAPK pathways. Int J Colorectal Dis 27: 1569-1578, 2012.

15. Livak KJ and Schmittgen TD: Analysis of relative gene expression data using real-time quantitative PCR and the 2(-Delta Delta C(T)) method. Methods 25: 402-408, 2001.

16. Xu CY, Liu SQ, Qin MB, Zhuge CF, Qin L, Qin N, Lai MY and Huang JA: SphK1 modulates cell migration and EMT-related marker expression by regulating the expression of p-FAK in colorectal cancer cells. Int J Mol Med 39: 1277-1284, 2017.
17. Nihira K, Miki Y, Iida S, Narumi S, Ono K, Iwabuchi E, Ise K Mori K, Saito M, Ebina M, et al: An activation of LC3A-mediated autophagy contributes to de novo and acquired resistance to EGFR tyrosine kinase inhibitors in lung adenocarcinoma. J Pathol 234: 277-288, 2014.

18. Miyamoto M, Takano M, Aoyama T, Soyama H, Yoshikawa $T$, Tsuda $\mathrm{H}$ and Furuya K: Inhibition of autophagy protein LC3A as a therapeutic target in ovarian clear cell carcinomas. J Gynecol Oncol 28: e33, 2017.

19. Giatromanolaki A, Koukourakis MI, Pouliliou S, Gatter KC, Pezzella F, Harris AL and Sivridis E: Overexpression of LC3A autophagy protein in follicular and diffuse large B-cell lymphomas. Hematol Oncol Stem Cell Ther 6: 20-25, 2013.

20. Martinez-Lopez N, Athonvarangkul D, Mishall P, Sahu S and Singh R: Autophagy proteins regulate ERK phosphorylation. Nat Commun 4: 2799, 2013.

21. Tanida I, Ueno T and Kominami E: LC3 and autophagy. Methods Mol Biol 445: 77-88, 2008.

22. Mowers EE, Sharifi MN and Macleod KF: Autophagy in cancer metastasis. Oncogene 36: 1619-1630, 2017.

23. Yu Y, Hou L, Song H, Xu P, Sun Y and Wu K: Akt/AMPK/mTOR pathway was involved in the autophagy induced by vitamin $\mathrm{E}$ succinate in human gastric cancer SGC-7901 cells. Mol Cell Biochem 424: 173-183, 2017.

24. Wang R, Shen Z, Yang L, Yin M, Zheng W, Wu B, Liu T and Song H: Protective effects of heme oxygenase-1-transduced bone marrow-derived mesenchymal stem cells on reducedsize liver transplantation: Role of autophagy regulated by the ERK/mTOR signaling pathway. Int J Mol Med 40: 1537-1548, 2017.

25. Nazio F and Cecconi F: Autophagy up and down by outsmarting the incredible ULK. Autophagy 13: 967-968, 2017.

26. Bao Y, Li K, Guo Y, Wang Q, Li Z, Yang Y, Chen Z, Wang J, Zhao $\mathrm{W}$, Zhang $\mathrm{H}$, et al: Tumor suppressor PRSS8 targets Sphk1/S1P/Stat3/Akt signaling in colorectal cancer. Oncotarget 7: 26780-26792, 2016.

27. González-Fernández B, Sánchez DI, Crespo I, San-Miguel B, Álvarez M, Tuñón MJ and González-Gallego J: Inhibition of the SphK1/S1P signaling pathway by melatonin in mice with liver fibrosis and human hepatic stellate cells. Biofactors 43: 272-282, 2017.

28. Gao Z, Wang H, Xiao FJ, Shi XF, Zhang YK, Xu QQ, Zhang XY, Ha XQ and Wang LS: SIRT1 mediates Sphk1/S1P-induced proliferation and migration of endothelial cells. Int J Biochem Cell Biol 74: 152-160, 2016.

29. Buchegger K, Silva R, López J, Ili C, Araya JC, Leal P, Brebi P, Riquelme I and Roa JC: The ERK/MAPK pathway is overexpressed and activated in gallbladder cancer. Pathol Res Pract 213: 476-482, 2017

30. Maeda Y, Oku M and Sakai Y: Autophagy-independent function of Atg8 in lipid droplet dynamics in yeast. J Biochem 161: 339-348, 2017.

31. Li L, Chen H, Gao Y, Wang YW, Zhang GQ, Pan SH, Ji L, Kong R, Wang G, Jia YH, et al: Long noncoding RNA MALAT1 promotes aggressive pancreatic cancer proliferation and metastasis via the stimulation of autophagy. Mol Cancer Ther 15: 2232-2243, 2016.

32. Kwon YT and Ciechanover A: The ubiquitin code in the ubiquitin-proteasome system and autophagy. Trends Biochem Sci 42: 873-886, 2017.

This work is licensed under a Creative Commons Attribution-NonCommercial-NoDerivatives 4.0 International (CC BY-NC-ND 4.0) License. 